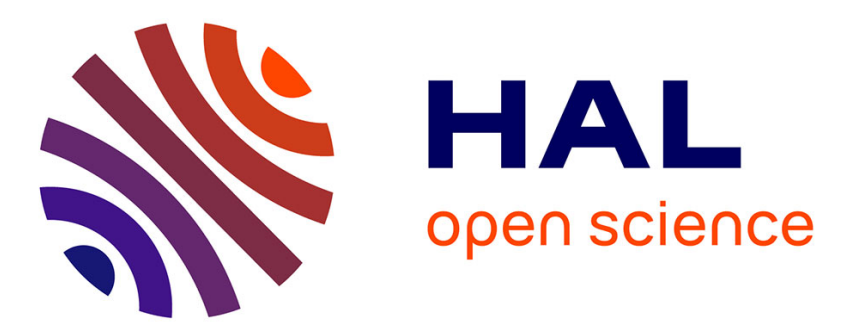

\title{
Femtosecond laser irradiation of dielectric materials containing randomly-arranged nanoparticles
}

Anton Rudenko, Jean-Philippe Colombier, Tatiana Itina

\section{To cite this version:}

Anton Rudenko, Jean-Philippe Colombier, Tatiana Itina. Femtosecond laser irradiation of dielectric materials containing randomly-arranged nanoparticles. Proceedings of SPIE, the International Society for Optical Engineering, 2016, 9737, pp.97370L. 10.1117/12.2217900 . ujm-01340988

\section{HAL Id: ujm-01340988}

\section{https://hal-ujm.archives-ouvertes.fr/ujm-01340988}

Submitted on 4 Jul 2016

HAL is a multi-disciplinary open access archive for the deposit and dissemination of scientific research documents, whether they are published or not. The documents may come from teaching and research institutions in France or abroad, or from public or private research centers.
L'archive ouverte pluridisciplinaire HAL, est destinée au dépôt et à la diffusion de documents scientifiques de niveau recherche, publiés ou non, émanant des établissements d'enseignement et de recherche français ou étrangers, des laboratoires publics ou privés. 


\title{
Femtosecond laser irradiation of dielectric materials containing randomly-arranged nanoparticles
}

\author{
Anton Rudenko, Jean-Philippe Colombier, and Tatiana E. Itina \\ Laboratoire Hubert Curien, UMR CNRS 5516, Lyon University, Bat. F, rue du Prof. Benoit \\ Lauras, 42000 Saint-Etienne, France
}

\begin{abstract}
We investigate femtosecond laser irradiation of dielectric materials containing randomly-arranged nanoparticles. For this, numerical modeling is performed based on three different methods: Mie theory, static solution of linear Maxwell's equations and a solution of nonlinear Maxwell's equations together with kinetic equations for free electron excitation/relaxation processes. First two approaches are used to define the static intensity distribution and to analyze the electromagnetic interaction between the nanoparticles. The third method allows us to investigate the complex dynamics of the laser-matter interaction. Multiphoton absorption is shown to be responsible for electron plasma generation in the regions of strong intensity enhancements in the vicinity of nanoparticles. The irradiation of the dielectric material leads to the elongation of nanoplasmas by the nearfield enhancement perpendicular to the laser polarization and to their strong interaction resulting in periodic arrangement. Numerical results shed light on such effects as femtosecond laser-induced nanograting formation.
\end{abstract}

Keywords: Multiphoton absorption, femtosecond-laser interaction, transparent materials, nonlinear Maxwell's equations, Mie theory

\section{INTRODUCTION}

Femtosecond laser interactions have attracted increasing interest due to new possibilities for the efficient nanostructuring of different materials. ${ }^{1-3}$ Nanoprocessing of transparent dielectrics is particularly promising since it enables writing optical waveguides, nanovoid arrays, polarization-dependent periodic surface structures and volume nanogratings. ${ }^{4,5}$ Recently, nanocomposites comprising metallic nanoparticles in a dielectric matrix have been shown to exhibit more pronounced nonlinear characteristics than the homogeneous transparent materials. These composites have already found a wide range of applications for solar cells, sensors and high-tech optical devices. ${ }^{6-10}$ In addition, periodic organization of nanostructures in transparent dielectrics containing metallic nanoparticles by ultrashort high-intensity laser irradiation has been evidenced in several recent works. ${ }^{11-14}$ Furthermore, the doping elements in glasses were found to significantly improve the quality and the smoothness of the nanostructures and to facilitate the accumulation processes responsible for the self-organization. ${ }^{14-17}$

The optical response of metallic nanoparticles embedded in a dielectric matrix was studied in many works. ${ }^{18-22}$ The dipole-dipole interaction between polarized nanoparticles was found to be among possible sources of the composite optical nonlinearity. Particularly, it was shown that the impact of the dipole-dipole interaction increased with rise of nanoparticle concentration in the composite. ${ }^{22}$ Until now, the effect of the dipole-dipole interactions on the optical characteristics of the nanocomposites was mostly studied by the discrete dipole approximation for simulating clusters of spheres. It is worth noting that the coupled dipole method, being a linear approximation, is suitable only for weak optical fields. Moreover, the method disregards the transient properties of laser-irradiated glass.

When a high-intensity laser beam is tightly focused inside transparent dielectric, the properties of the material change considerably due to photoionization and avalanche effects. ${ }^{23-26}$ Furthermore, metallic nanoparticles embedded in glass matrix were shown to enhance nonlinear absorption during the laser structuring process. ${ }^{13}$ The scenario of nanoplasmas growth due to localized inhomogeneous nonlinear ionization was discussed in several pioneer works. ${ }^{27-29}$ In particular, the nanoplasmonics model based on the near-field enhancement at the tip of the

\footnotetext{
E-mail: anton.rudenko@univ-st-etienne.fr, Telephone: +33 (0)7 82314452
} 
nanoplasmas was proposed as the mechanism of the nanogratings formation in glass. ${ }^{29}$ The generated electron plasma kinetics has been investigated in dielectrics in a few recent articles. ${ }^{30-35}$

Herein, we discuss the dynamics of high-intensity ultrafast laser interaction with nanocomposites. We start by considering the optical response and the nonlinear interaction with one nanoparticle. Then, we investigate the collective response of a periodically-arranged nanoparticle array. Finally, we show the results for randomly distributed inclusions embedded in glass matrix.

\section{NUMERICAL MODEL}

In this paper, we use different methods to analyze the optical response of nanoparticles in glass. Method A and Method B provide static intensity distribution. Method C describes the complex dynamics of laser-matter interaction with nanoparticles in glass.

The first method (Method A) is an analytical static solution and is based on an approximation of the Mie solution. $^{36,37}$ The scattered electric fields are calculated analytically for the case of a small $q \ll 1$ sphere in the near-field $k r \ll 1$ and in the far-field $k r \gg 1$ (see appendix). The total scattered field is the superposition of the fields scattered by each nanoparticle. The intensity corresponds to the square of the sum of the scattered and the incident fields. The approach describes the interference between the incident plane wave and the scattered field by homogeneous sub-wavelength spheres. The main advantage of this method is that we can separate dipoledipole interaction from other types of more complex interactions as well as the response of each individual dipole from the interparticle dipole coupling. Unfortunately, the approach is applicable only for the inhomogeneities of special shape and limited size.

The second method (Method B) solves the linear Maxwell's equations using finite-difference time-domain (FDTD) method. ${ }^{38}$ The method describes the intensity distribution at a fixed time of propagation and with a fixed density profile $n_{e}(x, z, t)=n_{e 0}(x, z)$. This approach still allows us to separate the contribution of each electric field and, therefore, the incident field and the scattered field from the total field. The method solves only static problems. Additionally, even at the fixed time, it is not precise because of the difficulty to define exactly the density profiles evolving in the study.

Finally, the third method (Method C) is based on the nonlinear Maxwell's equations coupled with electron density equation. The kinetics of the electron plasma in the transparent material is described, as follows

$$
\left\{\begin{array}{l}
\frac{\partial \vec{E}}{\partial t}=\frac{\nabla \times \vec{H}}{\epsilon_{0}}-\frac{1}{\epsilon_{0}} \vec{J}_{n l} \\
\frac{\partial \vec{H}}{\partial t}=-\frac{\nabla \times \vec{E}}{\mu_{0}} \\
\frac{\partial n_{e}}{\partial t}=\left(n_{a}-n_{e}\right) w_{p i}-\frac{n_{e}}{\tau_{t r}},
\end{array}\right.
$$

where $\vec{E}$ is the electric field, $\vec{H}$ is the magnetic field, $\vec{J}_{n l}$ is the nonlinear current consisting of two parts, the first term derived from the Drude model for the dispersive media with time-dependent electron density $n_{e}$ and the second photoionization term considering a complete Keldysh photoionization rate $w_{p i}{ }^{39}$ Free carrier number density changes due to photoionization $w_{p i}$ and recombination effect with $\tau_{t r}=150 \mathrm{fs} .{ }^{40}$ The electromagnetic response of the metallic nanoparticles is calculated from the linear Maxwell's equations with the current term derived from the Drude-Lorentz model for gold. ${ }^{41}$ In this method, we consider initial Gaussian electric field profile and Gaussian temporal envelope. The details of the numerical model and modeling parameters for glass can be found in Ref. [35]. This method is able to follow the kinetics of electron plasma generated in the near field of the embedded nanoparticles. However, the method does not allow us to separate linear and nonlinear processes and to elucidate which interaction is responsible for the nanoplasmas growth and interaction.

We note, that all the resulting snapshots of the electric fields, intensity and electron density are in the plane $x O z$, where the light is polarized along $x$ direction and $z$ is the propagation direction. 

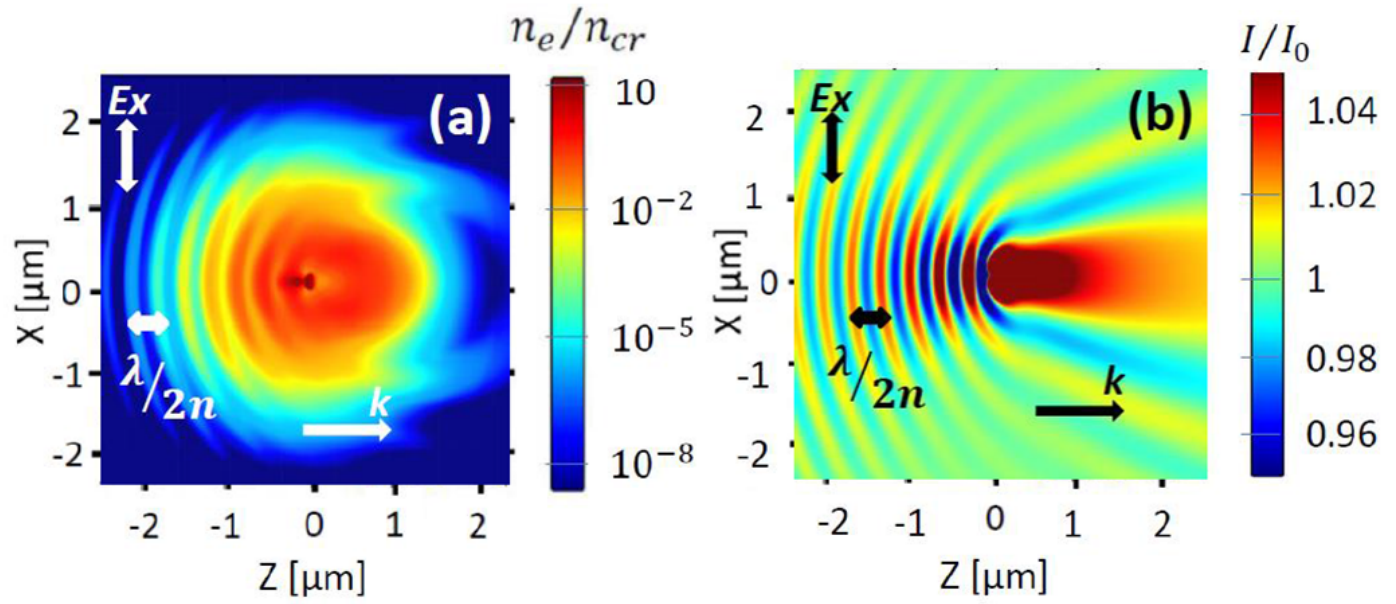

Figure 1. (a) Electron density distribution is calculated by 2D-Maxwell's equations coupled with electron density equation (Method C) at the peak of 80 fs laser pulse irradiating a single nanoparticle; (b) Static analytical Mie theory intensity distribution (Method A) for a small nanoparticle of radius $a=5 \mathrm{~nm}$ localized in $(0,0)$ (interference between the incident plane wave and the scattered spherical wave). Here, laser irradiation conditions: pulse duration $\theta=80 \mathrm{fs}$ (FWHM), irradiation wavelength in air $\lambda=800 \mathrm{~nm}$, pulse energy $E=300 \mathrm{~nJ}$. Electron density is normalized to its critical value at $800 \mathrm{~nm} n_{c r}=1.7 \times 10^{27} \mathrm{~m}^{-3}$ and $\vec{k}$ is the incident laser wave vector.

\section{RESULTS AND DISCUSSION}

First, we consider the optical response of one nanoparticle embedded in glass. The intensity in the near-field of the gold nanoparticle is strongly enhanced in the polarization direction and leads to the organization of electron plasma (non visible in Fig. 1(a)). ${ }^{33,35,42}$ The interference between the incident and the scattered farfield spherical wave leads to the organization of periodical minima and maxima in the direction of the light propagation (Fig. 1(a,b)). This phenomenon can be explained by considering the dipole approximation method (Method A). The scattered electric field $E_{x}^{(s)} \propto E_{\theta}^{(s)} \propto B_{1}^{e} \frac{\exp (i k z)}{k z}$ interferes with the incident polarization field $E_{x}^{(i)} \propto \exp (-i k z)$ (see appendix). Fig. 1(b) shows that the interference results in the organization of the standing wave with the intensity distribution $I \propto \frac{\exp (2 i k z)}{(k z)^{2}}$ having maxima and minima separated by $\Delta z=\lambda / 2 n$. The dynamics of the interaction of the laser light with plasma is defined by this strong enhancement in the direction backward to the laser propagation.

Second, we study the interaction of the organized nanoplasmas from several nanoparticles embedded in glass (Fig. 2). The mutual enhancement induced by the plasma interaction strongly depends on the distance between the initial nanoparticles. The optical response of the ensemble is the sum of the individual nanoplasma contributions modified by interparticle coupling that depends on specific geometry and, therefore, on the initial particle localization. Fig. 2(a) shows that for widely separated nanoparticles, the behavior is independent. Hence, the interference between the incident and the scattered field dominates over plasmonic modes. ${ }^{43}$ At closer distance, the interparticle coupling starts playing a role. We can see periodically organized patterns between the nanoplasmas corresponding to the mutual enhancements in Fig. 2(b,c). The closer the nanoplasmas are, the stronger their collective response. ${ }^{22}$ It is worth noting that the approximation method A cannot be applied in the case of closely separated nanoparticles with $k r \approx 1$. Thus, it is inapplicable for nanoparticles with interparticle separation significantly less than the irradiation wavelength in medium. The method B solving the complete system of Maxwell's equations should be used to understand the interaction of nanoplasmas in close proximity.

In the cases of one nanoparticle and widely separated nanoparticles embedded in glass, the resulting intensity distribution was defined mostly by the $E_{x}^{(s)} \propto E_{\theta}^{(s)} \propto B_{1}^{e} \frac{\exp (i k z)}{k z}$ electric field component, where $B_{1}^{e}$ is the first electric dipole moment (see appendix). The contribution of $E_{r}^{(s)} \propto B_{1}^{e} \frac{\exp (i k r)}{(k r)^{2}}$ is roughly negligible as it decreases very fast in the radial direction. However, for metallic nanoparticles, the magnetic moment is of the same order as 


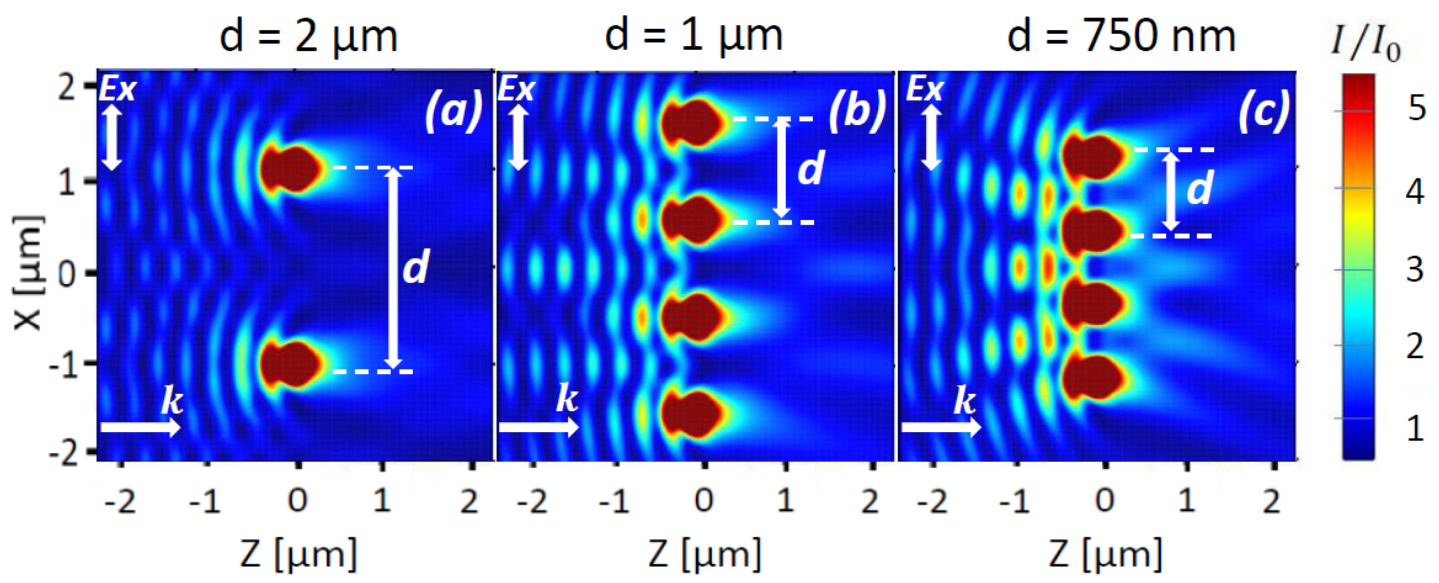

Figure 2. Static analytical Mie theory intensity distribution (Method A) for small nanoparticles of radius $a=50 \mathrm{~nm}$ with interparticle separations a) $d=2 \mu \mathrm{m}$ b) $d=1 \mu \mathrm{m}$ c) $d=750 \mathrm{~nm}$ (interference between the incident plane wave and the scattered spherical wave). Laser irradiation wavelength is $\lambda=800 \mathrm{~nm}$ in air.

the dipole moment order (see appendix). ${ }^{37,44}$ The orthogonal scattered field in the first approximation is defined as $E_{z}^{(s)} \propto E_{\theta}^{(s)} \propto B_{1}^{m} \frac{\exp (i k r)}{k r}$, where $B_{1}^{m}$ is the first magnetic dipole moment. The contribution of this field influences strongly the resulting intensity distribution. In the next paragraph we investigate the contribution of the orthogonal field.

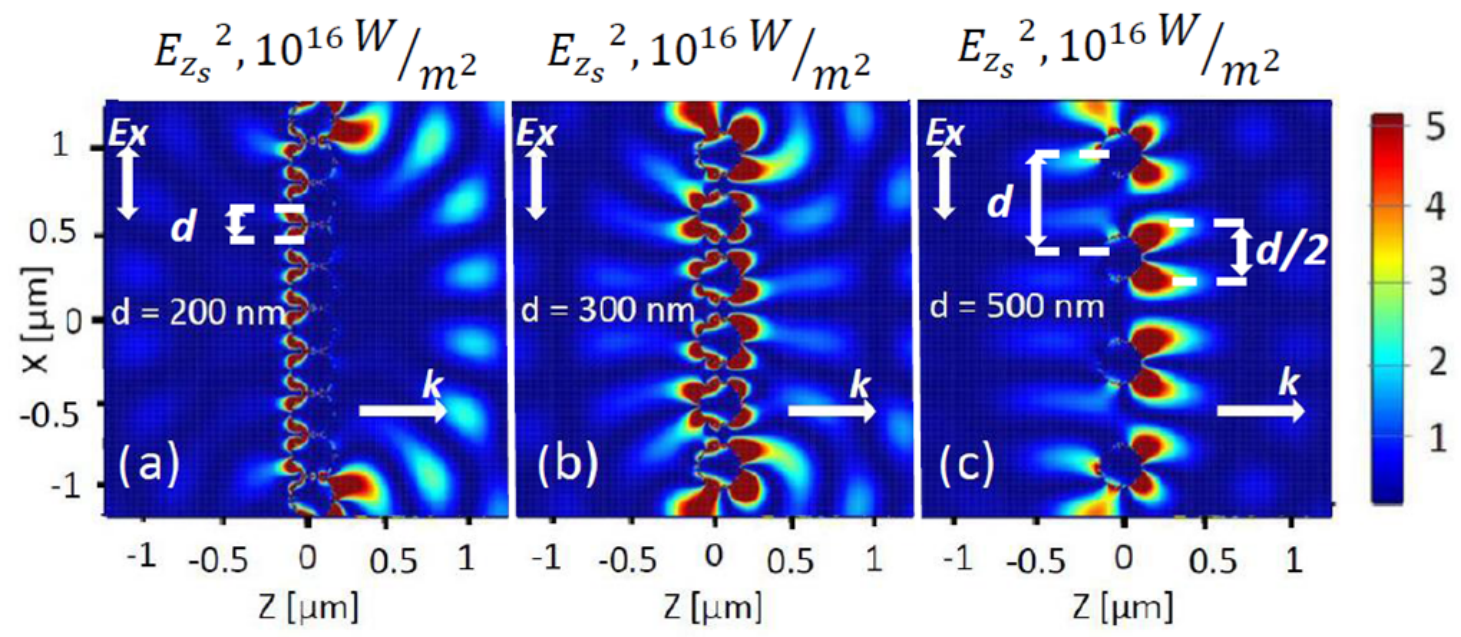

Figure 3. Multiple scattering intensity distributions for a periodic array of nanoparticles of radius $a=100 \mathrm{~nm}$ with different interparticle separations: (a) $d=200 \mathrm{~nm}$, (b) $d=300 \mathrm{~nm}$, (c) $d=500 \mathrm{~nm}$ are calculated by linear Maxwell's equations (Method B). Laser irradiation wavelength is $\lambda=800 \mathrm{~nm}$ in air.

In what follows, we analyze the character of multiple scattering induced by the orthogonal field from closely arranged nanoparticles (Fig. 3). An array of periodic high density spherical nanoparticles with radius $a=$ $100 \mathrm{~nm}$ is illuminated simultaneously by a plane wave. We vary the interparticle separation distance $d$ and study the resulting intensity distribution calculated by linear Maxwell's equations (Method B). For particle dimensions much smaller than the wavelength, interference effect is negligible but interparticle coupling becomes significant. For nearly touching nanoparticles, plasmons exhibit multipolar behavior, ${ }^{45}$ including a very high local concentration of electromagnetic energy in the vicinity of their conductive contact Fig. 3(a). In this 
case, the scattering patterns depend strongly on the particle size and, correspondingly, on the interparticle separation. Therefore, the period of the interference patterns due to multiple scattering is directly proportional to the interparticle separation. However, the near-field enhancement might have nontrivial distribution when the interparticle coupling effects take place. ${ }^{46,47}$ Fig. 3(b) demonstrates that as $d$ grows, the periodicity of enhancements also changes. For distances longer than the nanoparticle size, the character of scattering is qualitatively different and the periodicity is about half the interparticle separation in Fig. 3(c). It is worth noting that the intensity enhancement induced in the near-field of the closely spaced nanoparticles by the orthogonal field might considerably change the electron density distribution and the direction in which the nanoplasmas grow.

We have investigated the role of both components of the electric field in the case of periodically arranged nanoparticles without taking into account the transient properties of the dielectric material. We note, that such a physical interpretation can be considered only as a very rough approximation of the complex studying problem where the size and the density of nanoplasmas change in time. Hence, we perform FDTD calculations based on solution of the system of coupled equations (Method C) with initially periodically arranged nanoparticles (Fig. 4). Interestingly, the electron density snapshots taken before the pulse peak already show two regimes with different scattering behavior for different initial interparticle separations. In both cases, the nanoplasmas elongate from the nanoparticles in the backward propagation direction. For the nanoparticles in close proximity, the nanoplasmas merge together organizing the nanoplanes with exact periodicity of the initial interparticle separation in Fig. 4(a). At larger separation distances, each nanoplasma is divided into two and the final periodicity is half the interparticle separation in Fig. 4(b). In fact, such behavior can be explained by the intensity enhancements induced by the orthogonal field and calculated by linear Maxwell's equations in Fig. $3(\mathrm{c})$. The nanoplasmas always grow into the direction where the intensity is strongly enhanced.

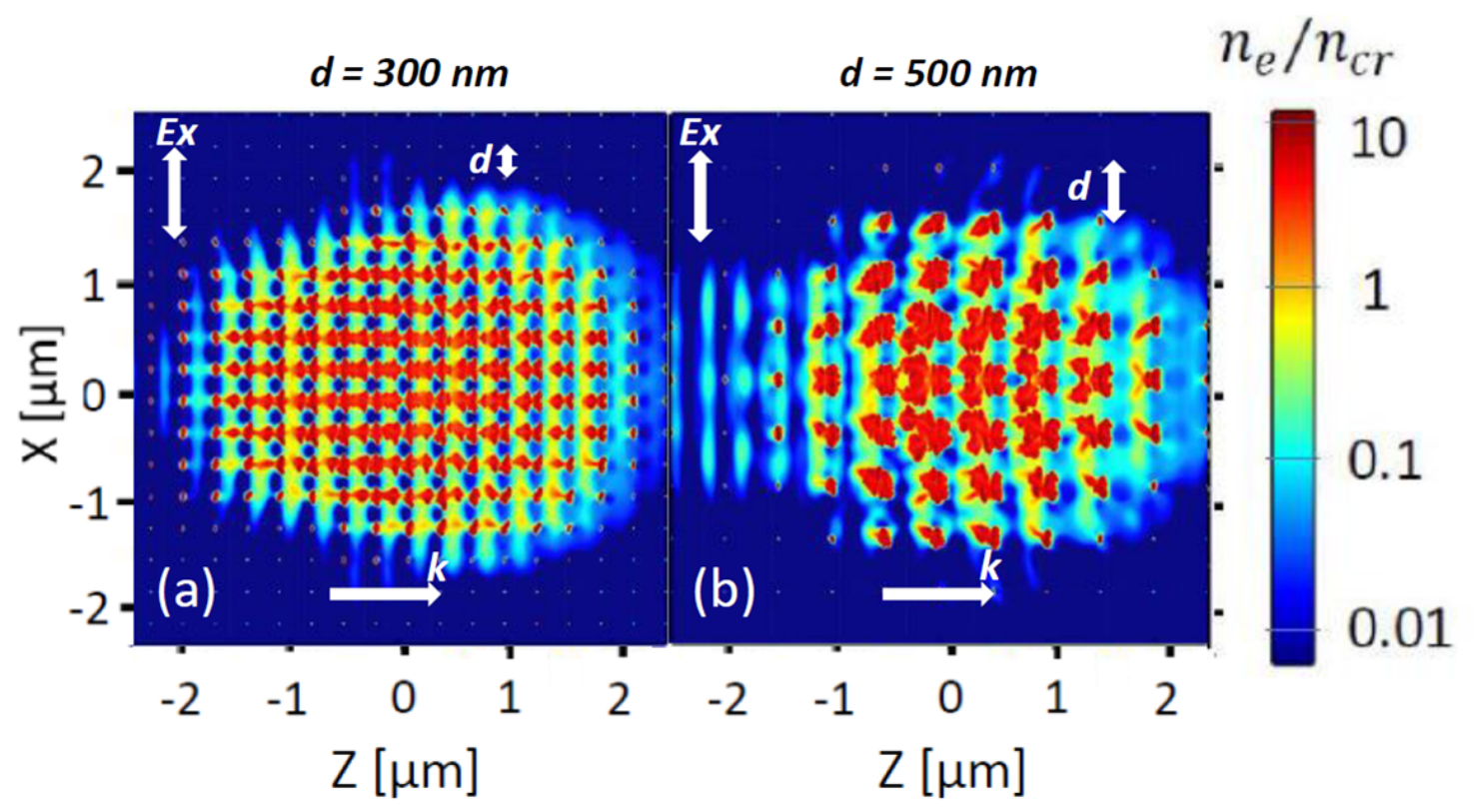

Figure 4. Electron density snapshots calculated by 2D-Maxwell's equations coupled with electron density equation (Method C) 40 fs before the pulse peak. Laser irradiation conditions: pulse duration $\theta=240 \mathrm{fs}$ (FWHM), irradiation wavelength in air $\lambda=800 \mathrm{~nm}$, pulse energy $E=500 \mathrm{~nJ}$. Electron density is normalized to its critical value at $800 \mathrm{~nm} n_{c r}=1.7 \times 10^{27} \mathrm{~m}^{-3}$. The interparticle separation is (a) $d=300 \mathrm{~nm}$, (b) $d=500 \mathrm{~nm}$.

Finally, the calculation results using nonlinear Maxwell's equations coupled with free electron density equation (Method C) for glass with initial randomly distributed nanoparticles are shown in Fig. 5. The first snapshot in Fig. 5(a) taken far before the peak pulse shows that each nanoparticle exhibits dipole behavior in the near-field and in the far-field. The interference of the incident and the scattered spherical waves from nanoparticles induces periodic strong enhancements backwards to the laser propagation. As a result, the nanoplasmas are elongated 
perpendicular to the laser propagation in Fig. 5(b). They strongly interact with each other. The nanoplasmas in close proximity merge together, slightly changing their direction. Fig. 5(c) shows that the mutual interaction leads to organization of quasi-periodic nanoplasma planes perpendicular to the laser polarization. Previously, it was reported that femtosecond laser irradiation of fused silica bulk might lead to the self-organization of nanogratings consisting of high density plasma. ${ }^{13,29,42,48,49}$ Small inhomogeneities are required to initiate the process. ${ }^{33}$ Here, we show that the nanoparticles can also play the same role of seeds for the nanoplasmas. Moreover, we underline that the self-organization of final structures can be explained by the electromagnetic approach coupled with the electron density equation to describe the dynamics of femtosecond laser interaction with dielectric materials.
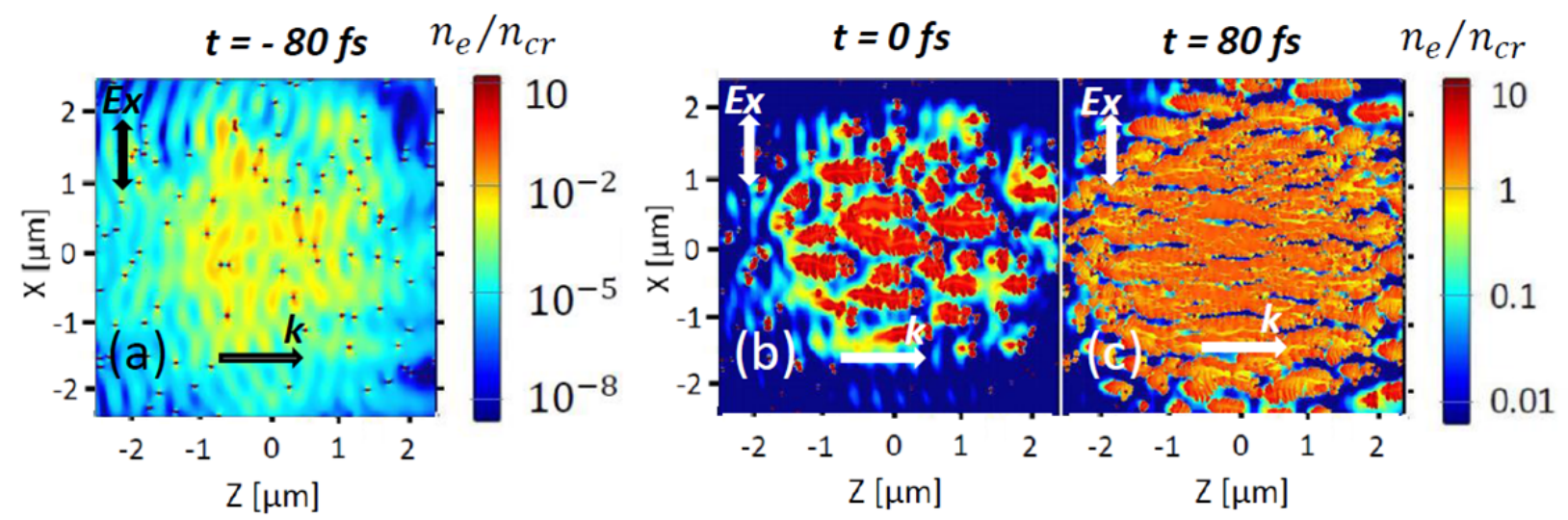

Figure 5. Electron density snapshots are calculated by 2D-Maxwell's equations coupled with electron density equation (Method C) revealing the evolution of nanogratings (a) 80 fs before the pulse peak, (b) at the pulse peak, (c) $80 \mathrm{fs}$ after the pulse peak. Laser irradiation conditions: pulse duration $\theta=240 \mathrm{fs}$ (FWHM), irradiation wavelength in air $\lambda=800$ $\mathrm{nm}$, pulse energy $E=500 \mathrm{~nJ}$. Electron density is normalized to its critical value at $800 \mathrm{~nm} n_{c r}=1.7 \times 10^{27} \mathrm{~m}^{-3}$. The average initial interparticle distance is $d=400 \mathrm{~nm}$. In Fig. 5(a) the scale is different than in Fig. 5(b) and in Fig. 5(c).

\section{CONCLUSION}

We have investigated ultrashort laser interaction with dielectric materials containing randomly-arranged nanoparticles. Three methods have been applied to explain the optical response of the quasi-metallic nanoplasma organized due to enhanced nonlinear absorption in the vicinity of the embedded nanoparticles: analytical solution from Mie theory (Method A), linear Maxwell's equations (Method B) and nonlinear Maxwell's equations coupled with electron density equation (Method C).

1) It is shown that the dipole approximation method can explain the near-field enhancement at the tip of nanoplasma resulting in nanoplasma growth.

2) The distance between the randomly elongated nanoplasmas should be at least less than the irradiation wavelength in glass, otherwise, the mutual enhancement is negligible. This distance allows us to estimate the initial concentration of the embedded nanoparticles which is required for significant near-field enhancement.

3) At closer nanoplasma separation, the mutual interaction exhibits complex behavior. Particularly, the contribution of the orthogonal field for the closely arranged metallic nanoparticles is not negligible. The intensity enhancement induced by the orthogonal field might considerably change the electron density distribution and the direction in which the nanoplasmas grow.

4) The results of calculations show that the nanoparticles act like reservoirs of available electrons, ${ }^{13}$ inducing the formation of long nanoplasmas. The nanoplasmas mutually interact and are arranged in quasi-periodic planes perpendicular to the laser polarization. 


\subsection{Appendix}

The electric fields scattered by homogeneous sphere in spherical coordinates $(r, \theta, \phi)$ can be written as follows ${ }^{36,37}$

$$
\left\{\begin{array}{l}
E_{r}^{(s)}=\frac{\cos \phi}{k^{2} r^{2}} \sum_{l=1}^{\infty} l(l+1) B_{l}^{e} \varsigma_{l}^{(1)}(k r) P_{l}^{(1)}(\cos \theta) \\
E_{\theta}^{(s)}=-\frac{\cos \phi}{k r} \sum_{l=1}^{\infty}\left(B_{l}^{e} \varsigma_{l}^{(1) \prime}(k r) P_{l}^{(1) \prime}(\cos \theta) \sin \theta-i B_{l}^{m} \varsigma_{l}^{(1)}(k r) P_{l}^{(1)}(\cos \theta) \frac{1}{\sin \theta}\right) \\
E_{\phi}^{(s)}=-\frac{\sin \phi}{k r} \sum_{l=1}^{\infty}\left(B_{l}^{e} \varsigma_{l}^{(1) \prime}(k r) P_{l}^{(1)}(\cos \theta) \frac{1}{\sin \theta}-i B_{l}^{m} \varsigma_{l}^{(1)}(k r) P_{l}^{(1) \prime}(\cos \theta) \sin \theta\right)
\end{array}\right.
$$

where $B_{l}^{e}$ and $B_{l}^{m}$ are electric and magnetic dipole moments, $k$ is the wave vector in the medium, $\varsigma_{l}^{(1)}(k r)$ are the spherical Henkel functions of the first kind, and $P_{l}^{(1)}(\cos \theta)$ are Legendre polynomials of the first kind. Additionally, the fields have a time dependence factor $\exp (-i \omega t)$ that has been suppressed in the above equations.

The asymptotes for Henkel functions are as follows

i) Near-field $(k r \ll 1)$ : $\varsigma_{1}^{(1)}(k r)=-\frac{i}{k r}$ and $\varsigma_{1}^{(1) \prime}(k r)=\frac{i}{(k r)^{2}}$.

ii) Far-field $(k r \gg 1): \varsigma_{1}^{(1)}(k r)=-\exp (i k r)$ and $\varsigma_{1}^{(1) \prime}(k r)=-i \cdot \exp (i k r)$.

Small size approximations $(q \ll 1, n q \ll 1, l=1)$ : $B_{1}^{e}=i q^{3} \frac{n^{2}-1}{n^{2}+2}, B_{1}^{m}=i q^{5} \frac{n^{2}-1}{30}, B_{l}^{e} \rightarrow 0, B_{l}^{m} \rightarrow 0, l>1$.

For small metallic nanoparticle $(q \ll 1, n q \gg 1, l=1)$ : $B_{1}^{e}=i q^{3}, B_{1}^{m}=i \frac{q^{3}}{2}, B_{l}^{e} \rightarrow 0, B_{l}^{m} \rightarrow 0, l>1$.

Legendre polynomials for $l=1: P_{1}^{(1)}(\cos \theta)=\sin \theta$ and $P_{l}^{(1) \prime}(\cos \theta)=-\frac{\cos \theta}{\sin \theta}$.

The scattered electric fields in Cartesian coordinate system:

$$
\left\{\begin{array}{l}
E_{x}^{(s)}=E_{r}^{(s)} \sin \theta \cos \phi+E_{\theta}^{(s)} \cos \theta \cos \phi-E_{\phi}^{(s)} \sin \phi \\
E_{y}^{(s)}=E_{r}^{(s)} \sin \theta \sin \phi+E_{\theta}^{(s)} \cos \theta \sin \phi+E_{\phi}^{(s)} \cos \phi \\
E_{z}^{(s)}=E_{r}^{(s)} \cos \theta-E_{\theta}^{(s)} \sin \theta .
\end{array}\right.
$$

\subsection{Acknowledgments}

This work was supported by the NANODIELEC project, LABEX MANUTECH SISE (ANR-10-LABEX-0075) of Université de Lyon, within the program Investissements d'Avenir (ANR-11-IDEX-0007) operated by the French National Research Agency (ANR).

\section{REFERENCES}

[1] Schaaf, P., [Laser Processing of Materials], Springer Series in Materials Science (2010).

[2] Thomson, R., Leburn, C., and Reid, D., [Ultrafast Nonlinear Optics], Springer International Publishing (2013).

[3] Sugioka, K. and Cheng, Y., "Ultrafast lasers - reliable tools for advanced materials processing," Light: Science 6 Applications 3, 1-12 (2014).

[4] Qiu, J., Miura, K., and Hirao, K., "Femtosecond laser-induced microfeatures in glasses and their applications," Journal of Non-Crystalline Solids 354(1213), 1100 - 1111 (2008). Proceedings of the 2005 International Conference on Glass.

[5] Tan, D., Sharafudeen, K. N., Yue, Y., and Qiu, J., "Femtosecond laser induced phenomena in transparent solid materials: Fundamentals and applications," Progress in Materials Science 76, $154-228$ (2016).

[6] Justin, T., Hernando, G., Radhakrishna, S., and Ramki, K., "In silico design of metall-dielectric nanocomposites for solar energy applications," Proc. SPIE 6648, 66480L.1-66480L.8 (2007).

[7] Pillai, S. and Green, M., "Plasmonics for photovoltaic applications," Solar Energy Materials and Solar Cells 94(9), 1481 - 1486 (2010). 
[8] Chen, Y., Karvonen, L., Säynätjoki, A., Ye, C., Tervonen, A., and Honkanen, S., "Ag nanoparticles embedded in glass by two-step ion exchange and their sers application," Opt. Mater. Express 1, 164-172 (Jun 2011).

[9] Petrov, M., [Glass-metal nanocomposites for photonics applications], Publications of the university of Eastern Finland, Dissertations in Forestry and Natural Sciences 101 (2013).

[10] Stratakis, E. and Kymakis, E., "Nanoparticle-based plasmonic organic photovoltaic devices," Materials Today 16(4), 133 - 146 (2013).

[11] Sendova, M., Sendova-Vassileva, M., Pivin, J. C., Hofmeister, H., Coffey, K., and Warren, A., "Experimental study of interaction of laser radiation with silver nanoparticles in sio2 matrix," Journal of Nanoscience and Nanotechnology 6, 748-755 (2006).

[12] Sendova-Vassileva, M., Sendova, M., Hofmeister, H., and Pivin, J. C., "Interaction of laser irradiation with au nanoparticles in sio2 thin films," Journal of optoelectronics and advanced materials 11, 1071-1076 (2009).

[13] Vangheluwe, M., Liang, F., Petit, Y., Hée, P., Ledemi, Y., Thomas, S., Fargin, E., Cardinal, T., Messaddeq, Y., Canioni, L., and Vallée, R., "Enhancement of nanograting formation assisted by silver ions in a sodium gallophosphate glass," Opt. Lett. 39, 5491-5494 (Oct 2014).

[14] Vangheluwe, M., Petit, Y., Liang, F., Hee, P., Ledemi, Y., Thomas, S., Fargin, E., Cardinal, T., Messaddeq, Y., Canioni, L., and Vallee, R., "Femtosecond laser-induced nanogratings formation assisted by silver ions in a gallophosphate glass and correlated optical properties," in [Photonics North, 2015], 1-6 (June 2015).

[15] Lancry, M., Poumellec, B., Chahid-Erraji, A., Beresna, M., and Kazansky, P. G., "Dependence of the femtosecond laser refractive index change thresholds on the chemical composition of doped-silica glasses," Opt. Mater. Express 1, 711-723 (Aug 2011).

[16] Umran, F. A., Liao, Y., Elias, M. M., Sugioka, K., Stoian, R., Cheng, G., and Cheng, Y., "Formation of nanogratings in a transparent material with tunable ionization property by femtosecond laser irradiation," Opt. Express 21, 15259-15267 (Jul 2013).

[17] Liao, Y. and Cheng, Y., "Femtosecond laser 3d fabrication in porous glass for micro- and nanofluidic applications," Micromachines 5(4), 1106 (2014).

[18] Cummings, K. D., Garland, J. C., and Tanner, D. B., "Optical properties of a small-particle composite," Phys. Rev. B 30, 4170-4182 (Oct 1984).

[19] Inouye, H., Tanaka, K., Tanahashi, I., and Hirao, K., "Ultrafast dynamics of nonequilibrium electrons in a gold nanoparticle system," Phys. Rev. B 57, 11334-11340 (May 1998).

[20] Grochowska, K., Sliwinski, G., Iwulska, A., Sawczak, M., Nedyalkov, N., Atanasov, P., Obara, G., and Obara, M., "Engineering au nanoparticle arrays nanoparticles on sio2 glass by pulsed uv laser irradiation," Plasmonics 8, 105-113 (2013).

[21] Lee, W. M., El-Ganainy, R., Christodoulides, D. N., Dholakia, K., and Wright, E. M., "Nonlinear optical response of colloidal suspensions," Opt. Express 17, 10277-10289 (Jun 2009).

[22] Panov, A. V., "Impact of interparticle dipole-dipole interactions on optical nonlinearity of nanocomposites," Journal of Modern Optics 60(11), 915-919 (2013).

[23] Stuart, B. C., Feit, M. D., Herman, S., Rubenchik, A. M., Shore, B. W., and Perry, M. D., "Nanosecondto-femtosecond laser-induced breakdown in dielectrics," Phys. Rev. B 53, 1749-1761 (Jan 1996).

[24] Kaiser, A., Rethfeld, B., Vicanek, M., and Simon, G., "Microscopic processes in dielectrics under irradiation by subpicosecond laser pulses," Phys. Rev. B 61, 11437-11450 (May 2000).

[25] Mao, S., Quéré, F., Guizard, S., Mao, X., Russo, R., Petite, G., and Martin, P., "Dynamics of femtosecond laser interactions with dielectrics," Applied Physics A 79(7), 1695-1709 (2004).

[26] Bourgeade, A., Mézel, C., and Saut, O., "Modeling the early ionization of dielectrics by ultrashort laser pulses," Journal of Scientific Computing 44(2), 170-190 (2010).

[27] Rajeev, P. P., Gertsvolf, M., Simova, E., Hnatovsky, C., Taylor, R. S., Bhardwaj, V. R., Rayner, D. M., and Corkum, P. B., "Memory in nonlinear ionization of transparent solids," Phys. Rev. Lett. 97, 253001 (Dec 2006).

[28] Rajeev, P. P., Gertsvolf, M., Hnatovsky, C., Simova, E., Taylor, R. S., Corkum, P. B., Rayner, D. M., and Bhardwaj, V. R., "Transient nanoplasmonics inside dielectrics," Journal of Physics B: Atomic, Molecular and Optical Physics 40(11), S273 (2007). 
[29] Taylor, R., Hnatovsky, C., and Simova, E., "Applications of femtosecond laser induced self-organized planar nanocracks inside fused silica glass," Laser \& Photonics Reviews 2(1-2), 26-46 (2008).

[30] Popov, K. I., McElcheran, C., Briggs, K., Mack, S., and Ramunno, L., "Morphology of femtosecond laser modification of bulk dielectrics," Opt. Express 19, 271-282 (Jan 2011).

[31] Bulgakova, N. M., Zhukov, V. P., and Meshcheryakov, Y. P., "Theoretical treatments of ultrashort pulse laser processing of transparent materials: toward understanding the volume nanograting formation and quill writing effect," Applied Physics B 113, 437-449 (2013).

[32] Gulley, J. R. and Lanier, T. E., "Model for ultrashort laser pulse-induced ionization dynamics in transparent solids," Phys. Rev. B 90, 155119 (Oct 2014).

[33] Buschlinger, R., Nolte, S., and Peschel, U., "Self-organized pattern formation in laser-induced multiphoton ionization," Phys. Rev. B 89, 184306 (May 2014).

[34] Hoyo, J. D., de La Cruz, A. R., Grace, E., Ferrer, A., Siegel, J., Pasquazi, A., Assanto, G., and Solis, J., "Rapid assessment of nonlinear optical propagation effects in dielectrics," Scientific Reports 5, 7650 (Jan. 2015).

[35] Rudenko, A., Colombier, J.-P., and Itina, T., "Femtosecond laser irradiation of fused silica with a nanometric inhomogeneity," in [Proc. of the 36th Progress in Electromagnetics Research Conference (PIERS), Prague, 2015], Proc. of the 36th Progress in Electromagnetics Research Conference (PIERS) , 1870-1876, The Electromagnetics Academy (July 2015).

[36] Mie, G., "Beiträge zur optik trüber medien, speziell kolloidaler metallösungen," Annalen der Physik 330(3), 377-445 (1908).

[37] Born, M. and Wolf, E., [Principles of Optics], Cambridge University Press, 7 ed. (1999).

[38] Yee, K. S., "Numerical solution of initial boundary value problems involving maxwells equations in isotropic media," IEEE Trans. Antennas and Propagation, 302-307 (1966).

[39] Keldysh, L. V., "Ionization in the field of a strong electromagnetic wave," Soviet Physics JETP 20, 13071314 (May 1965).

[40] Martin, P., Guizard, S., Daguzan, P., Petite, G., D'Oliveira, P., Meynadier, P., and Perdrix, M., "Subpicosecond study of carrier trapping dynamics in wide-band-gap crystals," Phys. Rev. B 55, 5799-5810 (Mar 1997).

[41] Rakić, A. D., Djurišić, A. B., Elazar, J. M., and Majewski, M. L., "Optical properties of metallic films for vertical-cavity optoelectronic devices," Appl. Opt. 37, 5271-5283 (Aug 1998).

[42] Liao, Y., Ni, J., Qiao, L., Huang, M., Bellouard, Y., Sugioka, K., and Cheng, Y., "Formation of nanogratings in a porous glass immersed in water by femtosecond laser irradiation," in [Proc. SPIE], 9350, 93500, Optical Society of America (2015).

[43] Rahmani, M., Miroshnichenko, A. E., Lei, D. Y., Luk'yanchuk, B., Tribelsky, M. I., Kuznetsov, A. I., Kivshar, Y. S., Francescato, Y., Giannini, V., Hong, M., and Maier, S. A., "Beyond the hybridization effects in plasmonic nanoclusters: Diffraction-induced enhanced absorption and scattering," Small 10(3), 576-583 (2014).

[44] Chapuis, P.-O., Laroche, M., Volz, S., and Greffet, J.-J., "Near-field induction heating of metallic nanoparticles due to infrared magnetic dipole contribution," Phys. Rev. B 77, 125402 (Mar 2008).

[45] Romero, I., Aizpurua, J., Bryant, G. W., and Abajo, F. J. G. D., "Plasmons in nearly touching metallic nanoparticles: singular response in the limit of touching dimers," Opt. Express 14, 9988-9999 (Oct 2006).

[46] Xu, H., Bjerneld, E. J., Aizpurua, J., Apell, P., Gunnarsson, L., Petronis, S., Kasemo, B., Larsson, C., Hook, F., and Kall, M., "Interparticle coupling effects in surface-enhanced raman scattering," Proc. SPIE 4258, 35-42 (2001).

[47] You, Y., Du, C., Ma, Y., Kasim, J., Yu, T., and Shen, Z., "Effect of near-field coupling on far-field inelastic scattering imaging of gold nanoparticles," Nanotechnology 19(39), 395705 (2008).

[48] Shimotsuma, Y., Kazansky, P. G., Qiu, J., and Hirao, K., "Self-organized nanogratings in glass irradiated by ultrashort light pulses," Phys. Rev. Lett. 91, 247405 (Dec 2003).

[49] Kazansky, P. G., Yang, W., Bricchi, E., Bovatsek, J., Arai, A., Shimotsuma, Y., Miura, K., and Hirao, K., "'quill" writing with ultrashort light pulses in transparent materials," Applied Physics Letters 90(15), (2007). 